\title{
Importancia y beneficios del parto humanizado
}

\section{Importance and benefits of humanized delivery}

\section{Importância e benefícios do parto humanizado}

\author{
Mariela G. Macías-Intriago I \\ mariela.macias@gmail.com \\ Javier I. Haro-Alvarado II \\ javier.haro@hotmail.com \\ Felicita E. Piloso-Gómez III \\ felicita.pilo@gmail.com
}

\author{
Gabriela L. Galarza-Soledispa IV \\ gabriela-galarza@gmail.com \\ María del Carmen Quishpe-Molina V \\ maria-carquis@yahoo.com \\ Brenda N. Triviño-Vera ${ }^{\mathrm{VI}}$ \\ brenda.trivi@hotmail.com
}

Recibido: 27 de abril de 2018 * Corregido: 18 de junio de $2018 *$ Aceptado: 08 de julio de 2018

I. Doctor en Medicina y Cirugía, Mg en Epidemiologia; Directora Distrital de Salud 13D01 Portoviejo; Docente de la UTM, Jipijapa, Ecuador.

II. Doctor en Medicina y Cirugía, Especialista en Auditoria Médica, Diplomado en Ciencias de la Educación para la Salud, Magister en Medicina Tropical, Docente de la UTM, Jipijapa, Ecuador.

III. Magister en Ciencia de Alimentos, Analista de Nutrición 2 Distrito de Salud 13D02, Portoviejo, Ecuador.

IV. Licenciada en Enfermería, Enfermera de Cuidado Directo Área Emergencia, Hospital Básico Jipijapa, Ecuador.

v. Médico General, Residente Hospital General Latacunga, Servicio Gineco Obstetricia, Latacunga, Ecuador.

VI. Obstetra, Hospital Básico Jipijapa, Jipijapa, Ecuador. 


\section{Resumen}

En el presente trabajo está basado en explorar la importancia y beneficios del parto humanizado, en que la mujer debe presentar el plan de parto que es un documento donde la mujer expresa sus preferencias, necesidades, deseos y expectativas sobre el proceso del parto y nacimiento. Esto incluye quiénes deben estar presentes, dónde debe ocurrir, cómo le gustaría que sea el ambiente, cual es la posición de su preferencia, qué métodos de alivio del dolor desea usar o no desea, qué quisiera que se haga o deje de hacer a su recién nacido. Que se respeten las 40 semanas del proceso del parto humanizado, mismo que brinda momentos emotivos para la madre con el contacto piel a piel con su hijo. Tan pronto nace el bebé, el equipo médico lo adapta a su abdomen para que ella pueda sentirlo inmediatamente, también se presta asistencia a la madre para la lactancia del niño, lo cual estrecha el vínculo entre ambos y se favorece desde las primeras horas del nacimiento el traspaso de los nutrientes a su hijo para su crecimiento y desarrollo. Los planes de parto influyen positivamente durante el proceso y culminación del parto humanizado. En Ecuador hasta ahora se aprueba la Ley de Parto Humanizado afín de reducir la alarmante tasa de cesáreas. Además para que las mujeres que buscan quedarse embarazadas, que ya lo están o que ya son madres, estén bien informadas del respeto a sus derechos sexuales, reproductivos y como usuarias del Sistema Nacional de Salud, que conozcan las recomendaciones de la OMS y del MSP sobre buenas prácticas. No pecar de ignorancia sobre lo que es un parto respetado, en que la mujer es la protagonista de su embarazo y parto, que se respeten sus tiempos, donde le brinden toda la información necesaria y las opciones para que tenga libertad de elección.

Palabras Claves: Plan de parto, importancia, 40 semanas, parto humanizado, contacto piel a piel, beneficios, respeto a sus derechos, Ley de Parto Humanizado. 


\begin{abstract}
In the present work, it is based on exploring the importance and benefits of humanized childbirth, in which the woman must present the birth plan, which is a document where the woman expresses her preferences, needs, wishes and expectations about the birth process and birth. This includes who should be present, where it should occur, how you would like the environment to be, what is your preferred position, what pain relief methods you want to use or do not want, what you would like done or not done to your newborn. That the 40 weeks of the humanized delivery process be respected, which provides emotional moments for the mother with skin-to-skin contact with her child. As soon as the baby is born, the medical team adapts it to her abdomen so that she can feel it immediately, the mother is also assisted in the breastfeeding of the child, which strengthens the bond between the two and is favored from the first hours of birth the transfer of nutrients to your child for growth and development. The plans of childbirth have a positive influence during the process and culmination of humanized delivery. In Ecuador, until now, the Humanized Delivery Act has been approved in order to reduce the alarming cesarean rate. Also for women who seek to become pregnant, who already are or who are already mothers, are well informed of respect for their sexual and reproductive rights and as users of the National Health System, who know the recommendations of the WHO and the MSP on good practices. Do not sin of ignorance about what is a respected delivery, in which the woman is the protagonist of her pregnancy and delivery, that her times are respected, where they provide all the necessary information and options so that she has freedom of choice.
\end{abstract}

Keywords: Birth plan, importance, 40 weeks, humanized delivery, skin-to-skin contact, benefits, respect for their rights, Humanized Delivery Act. 


\section{Introducción.}

El parto es el proceso natural en que los bebés nacen, es uno de los eventos más importantes en la vida de la mujer, en que no solo es nuestro derecho sino también nuestra responsabilidad de brindar todos los beneficios, por lo que el ofrecimiento del servicio sanitario precisa cuidados y atenciones especiales, dando a los protagonistas de este la importancia que demandan.

Históricamente, las mujeres han dado a luz en compañía de otras mujeres experimentadas de su familia o comunidad. Esta costumbre, a cargo de las llamadas parteras tradicionales o comadronas, sigue siendo habitual en muchas poblaciones indígenas de América Latina y de otras partes del mundo. Las altas tasas de mortalidad materna y neonatal que persisten entre estas poblaciones han sido atribuidas en ocasiones a lo que se considera un apoyo informal por parte de estas personas. En realidad, suelen ser consecuencia de la exclusión económica y social y de la ausencia de condiciones de higiene adecuadas, personal médico cercano o acceso a servicios de emergencia que puedan prevenir o aminorar posibles complicaciones. Johannsen, (2016).

En el periodo clásico y medieval, los médicos centraron sus intereses en el conocimiento teórico de la procreación, actuando solo en casos excepcionales, como cuando se necesitaba desmembrar a algún feto muerto para sacarlo del útero. En el siglo XVII cambio de rumbo pues se incorporaron cirujanos en la atención al parto y se dio inicio a la instrumentación con el fórceps (inventado por la familia Chamberlain), dando paso, de este modo, a la tecnificación de la asistencia en el nacimiento. Biurrun, (2013).

\footnotetext{
395 Vol. 4, núm. 3, julio 2018, pp. 392-415

Mariela G. Macías-Intriago; Javier I. Haro-Alvarado; Javier I. Haro-Alvarado; Gabriela L. GalarzaSoledispa; María del Carmen Quishpe-Molina; Brenda N. Triviño-Vera
} 
En cuanto al parto medicalizado, que es donde se da la intervención a través de tecnologías, medicamentos, y procedimientos empezó a darse en la revolución industrial en el siglo XIX, el inicio de la medicalización fue darle seguridad al parto sin embargo el autor resalta que se han ido incorporando procedimientos que ya se han convertido en un ritual del parto, configurándose muchas veces un hecho despersonalizado. Alonso y Gerard, (2009).

A mitad del siglo XX se institucionalizó el proceso de parto, pasando de los partos domiciliarios a los partos hospitalarios. Al considerarse el parto un proceso hospitalario, se incluyeron ciertas prácticas rutinarias y protocolizadas, como la episiotomía, el rasurado, los enemas, la estimulación del parto, sin estar avalado el uso rutinario de las mismas por la evidencia científica. Se incluyó el parto en el modelo sanitario de enfermedad. Maroto, et al. (2004).

En este momento, el proceso de nacimiento abandonó el ámbito de la tradición humanista para incluirse en el ámbito de la medicina especializada, tratando a la gestante como una mujer enferma que requiere asistencia médica. Biurrun, y Goberna, (2013).

En respuesta a ello, la Organización Mundial de la Salud (OMS) en el año 1985 realizó un encuentro en Brasil donde surgió la declaración "El nacimiento no es una enfermedad", que dio inicio al proceso de transformación del modelo de atención del parto, lo que actualmente se conoce como parto humanizado. Prolhuna, (2014).

Con las recomendaciones de la OMS sobre el nacimiento, se inicia un proceso de "normalización del parto", se insta a los estados a revisar la tecnología aplicada a los partos, y reconoce que cada mujer debe elegir el tipo de parto que desea, contribuyendo de este modo a devolver el protagonismo a la mujer. OMS, (1985) 
El parto inducido se desarrolla a partir de un enema que se le practica a la paciente, aplicándosele luego una serie de inyecciones hasta provocar el comienzo del trabajo de parto. Entonces se anestesia a la mujer, para presentarle, hora más tarde, cuando ella despierta medio confusa, dolorida e indiferente, su hijo desconocido bien lavado y vestido con su mejor ropa. Lange, (1988).

En 1996, se publica la guía “Cuidados en el parto normal: una guía práctica” elaborada por la Organización Mundial de la Salud en Ginebra. OMS, (1996).

La importancia de los Planes de Parto y Nacimiento radica en el respeto al Principio Bioético de Autonomía, aumentando de este modo el control que tienen las mujeres sobre el proceso del parto, contribuyendo a producir un efecto positivo sobre la satisfacción. Whitford, y Hillan, (1998).

Las diferentes visiones de atención que tienen los profesionales sanitarios del área obstétrica, se han generado polémicas al encontrarnos, aún hoy en día, con defensores de la actuación tradicional basada en una atención medicalizada. Biurrun, y Goberna, (2013).

La implantación a nivel nacional e internacional de este nuevo modelo es un hecho y requiere cambios en la praxis profesional, necesitándose la creación de protocolos o guías de práctica clínica consensuadas que detallen una forma de actuación humanizada de asistencia al parto. Biurrun y Goberna, (2013) y Martínez, (2011).

La tasa de cesáreas es considerada un indicador de buena práctica clínica al relacionarse con el buen control del embarazo y con una correcta asistencia al parto. OMS, (2015), Hernández, et al. (2014). 
En general, el porcentaje de cesáreas practicadas está más vinculado con el tipo de asistencia sanitaria que ofrece el profesional sanitario que a comorbilidades o complicaciones materno-fetales. OMS, (2015).

Ecuador tiene una tasa promedio de $41 \%$ de cesáreas que representa un incremento de aproximadamente el $60 \%$ en un promedio de 8 años. Otros países como Brasil y México tienen tasas aún más altas de cesáreas, de hasta el $80 \%$. En este entorno, una atención participativa, humana y respetuosa del parto requiere un esfuerzo adicional por parte de las embarazadas en términos de información, tiempo, costo y valor. De todas formas, las embarazadas tienen menos opciones reales en el sector público que en el privado de tener un parto humanizado. Las pacientes del sector privado pueden escoger su obstetra de confianza que atiende de la manera que prefieren y quien esta presente en el momento de dar a luz; mientras que en el sector público, la dinámica de la atención es menos personalizada y no se cuenta con la posibilidad de la libre elección. Johannsen, (2016).

\section{Metodología.}

El presente trabajo de investigación científico tiene una visión específica, se hizo la revisión de literatura más próxima. Para la realización del actual estudio, se consideró, información confiable sobre trabajos figurados, versados en este tema, se utilizaron informaciones bibliográficas, se la obtuvo de libros electrónicos, consultas electrónicas, revistas y artículos científicos.

\section{Desarrollo.}

Importancia del parto humanizado 
Bajo el lema "40 semanas Respetemos los tiempos del nacimiento", el Parto Respetado que tiene el objetivo de promover la modalidad del parto humanizado ante prácticas que son antinaturales y que pueden violentar a la mujer y al bebé. El parto es un momento transformador en la vida de una familia, sobre todo en el de la mujer y el niño que está por nacer. El parto respetado refiere a una modalidad de atención que contempla el respeto a los derechos de los padres y e hijos por nacer. Gago, (2017).

El parto humanizado o parto respetado es una modalidad de atención del parto caracterizada por el respeto a los derechos de los padres y los niños en el momento del nacimiento. Considera los valores de la mujer, sus creencias, y sentimientos respetando su dignidad y autonomía durante el proceso de dar a luz. Este tipo de partos reduce los nacimientos sobremedicados, empoderando a las mujeres y los usos de prácticas de maternidad basados en la evidencia como estrategia para humanizar el parto. Johannsen, (2016).

El Parto Humanizado implica que el nacimiento de un bebé debe ser una experiencia segura y positiva para toda la familia. El parto debe ser tratado como un proceso natural, con la madre en el centro de la toma de decisiones y sólo se debe intervenir cuando el equipo de salud así lo disponga por situaciones que así lo requieran. OMS, (2018).

Esta perspectiva tiene dos lados: el punto de vista de la madre o la pareja y el del bebé. Desde el punto de vista del bebé, para mí, uno de los pioneros del parto humanizado es el obstetra francés Frederick Leboyer. Ya en 1975 publicó su libro "Nacimiento sin violencia" que fue muy provocador en su época. Revolucionó la forma de atender partos en Europa, y lo sigue haciendo en el mundo. Leboyer se coloca en la perspectiva del bebé y describe las posibles percepciones y sentimientos del feto antes, durante y después de nacer. Johannsen, (2016). 
El parto humanizado, es el procedimiento en donde se priorizan las necesidades de la paciente y se reconoce a los padres como los protagonistas del proceso para que el personal de salud realice las mínimas intervenciones. Asimismo, se involucra a la pareja y a la familia en el nacimiento del bebé. En la Unidad de Cuidado Obstétrico donde la paciente es atendida con el principio TPR, es decir, que ese espacio está acondicionado para realizar el trabajo de parto, parto y recuperación tanto de la madre como de su hijo. Calderón, (2017).

Todavía hoy existe cierto desconocimiento de las prácticas médicas, que suele traducirse en temor. La herramienta fundacional del parto respetado es el vínculo que se establece entre la pareja y el equipo que la asistirá. Un vínculo atravesado por la capacidad de escucha, el intercambio de información, el diálogo y el afecto. Así, la pareja estará preparada tanto en los aspectos físicos como emocionales, para participar activamente en el nacimiento de su hijo. Gago, (2017).

"El parto normal es lo más fisiológico, posee menos complicaciones, y la madre queda más capacitada para atender a su hijo recién nacido y hacer sus cosas", afirma el Dr. Hott, quien además reconoce que el apego del bebé con la madre es mucho mayor en un parto normal. En definitiva, hay muchos menos riesgos, lo que se explica en las ventajas que este proceso natural conlleva al dar a luz a través del canal de parto normal, con todo lo que su desarrollo significa. Hott, (2016).

Un buen equipo de trabajo podrá ponerse en una situación humilde y escuchar lo que se necesita para poder actuar. Las parteras trabajamos activamente en construir y sostener ese vínculo, porque somos las principales encargadas de contener y acompañar a la embarazada y somos el nexo con los médicos. Si sabemos los deseos de cada paciente, podremos brindar la información correcta al obstetra. La mujer tiene derecho a saber sobre los procedimientos posibles para dar a luz, los 
pasos a seguir durante el parto y una explicación justa ante la indicación de determinada práctica, como puede ser una cesárea. Así, puede tomar decisiones seguras e informadas. Gago, (2017).

Parto humanizado, es el proceso de atención de parto en el que se busca respetar las opiniones y necesidades emocionales de la mujer y su familia como protagonistas del parto, además de adoptar medidas que sean beneficiosas, evitando prácticas intervencionistas innecesarias con la finalidad de crear un momento especial y en condiciones de dignidad humana. Vela, (2015),

Es a raíz de la Conferencia Internacional sobre la Humanización del Parto que fue organizada por integrantes del movimiento brasileño para la humanización, apoyada por el proyecto Luz, promovida por la Agencia de Cooperación Internacional de Japón y celebrada en el 2000 en Brasil, que nace el concepto de "humanización", el cual se define como "un proceso de comunicación y cuidado entre las personas que lleva a la auto transformación y la comprensión del espíritu fundamental de la vida y a un sentido de compasión y unidad con el universo, el espíritu y la naturaleza, los miembros de la familia, la comunidad, el país y la sociedad global, y también con otras personas en el futuro, así como con las generaciones pasadas". A partir de aquel entonces, diferentes autores comienzan a hacerse eco en sus artículos con dicho concepto. Biurrun, y Goberna, (2013).

Esto le lleva a proponer métodos más suaves y sensibles de atender partos, con el fin de reducir el estrés del bebé al pasar de un mundo acuático, oscuro y tranquilo al mundo aeróbico, luminoso y ruidoso, como por ejemplo: bajar las luces y las voces en la sala de parto, poner el bebé piel a piel con su madre inmediatamente después de nacer, cortar el cordón umbilical después de unos minutos para permitir una transición paulatina de la respiración umbilical a la pulmonar, y ofrecer un masaje cariñoso o un baño caliente y relajante al recién nacido. Johannsen, (2016).

\footnotetext{
401 Vol. 4, núm. 3, julio 2018, pp. 392-415
Mariela G. Macías-Intriago; Javier I. Haro-Alvarado; Javier I. Haro-Alvarado; Gabriela L. GalarzaSoledispa; María del Carmen Quishpe-Molina; Brenda N. Triviño-Vera
} 
El parto humanizado se basa en procedimientos obstétricos que procuran evitar las rutinas asistenciales sistematizadas. Y en la posición específica de la mujer para parir, cada una asume libremente aquella que le resulte más eficaz, cómoda o placentera durante todo el trabajo de parto y el período expulsivo. Indudablemente las preferencias espontáneamente referidas por la mujer, serán a favor de las posiciones verticales, acuclilladas, semisentadas o sentadas, de rodillas o de pie sostenidas por su pareja; con un fuerte rechazo a acostarse. En la búsqueda de la posición sienten claramente que el esfuerzo de pujar espontáneamente es más fácil, experimentando menos dolor sacrolumbar. Burgo, (2003).

El Parto normal es aquel parto que ocurre a través del canal de parto normal, que es la vagina. La operación cesárea es una intervención quirúrgica donde se abre el abdomen y el útero para la extracción del feto. Hott, (2016).

En los últimos años se está produciendo un cambio más que evidente en el modelo de atención sanitaria al parto, aumentando la importancia del concepto de humanización relacionado principalmente con la atención sanitaria ofrecida por los profesionales durante el trabajo de parto. Biurrun, y Goberna, (2013). Silva, et al. (2011).

Libros como “An Easier Childbirth - A Mother's Guide for Birthing Normally” o en español "Un nacimiento más fácil - Guía para madres sobre cómo dar a luz de forma normal" de Gayle Peterson son una ayuda valiosa para el empoderamiento de la mujer en este sentido, ya que ofrecen una preparación práctica, mental y emocional para el parto. Aspecto que es igual o, quizá, hasta más importante que la preparación física y logística. Johannsen, (2016).

\footnotetext{
402 Vol. 4, núm. 3, julio 2018, pp. 392-415

Mariela G. Macías-Intriago; Javier I. Haro-Alvarado; Javier I. Haro-Alvarado; Gabriela L. GalarzaSoledispa; María del Carmen Quishpe-Molina; Brenda N. Triviño-Vera
} 
Alonso y Gerard (2009), en su artículo denominado “El parto humanizado como herramienta para la prevención de la mortalidad materna y la mejora de la salud materno infantil", expone que el parto humanizado se basa en tres ideas fundamentales: la primera es que el parto y nacimiento son procesos fisiológicos que por naturaleza la mujer tiene su cuerpo preparado y capacitado, por lo tanto la mujer debe ser la protagonista; la segunda idea es que las herramientas médicas de atención al embarazo y parto son necesarias para salvaguardar la vida y se debe utilizar sólo en caso de emergencia, la tercer idea se refiere a la capacitación y conciencia que deben tener las mujeres para ser madres, ya que la experiencia del parto debe ser satisfactoria.

La idea de humanización en el parto se basa en el respeto a los derechos humanos, los derechos reproductivos y sexuales de las mujeres, sus parejas y, en general, de sus familias; su objetivo es cambiar la idea de que el embarazo y el parto son patologías que necesitan de intervención médica, y trata de recuperar la naturaleza del proceso procreativo como un hecho trascendental, íntimo, personal y memorable. Fundación Juan Vives Suriá, (2012).

Un parto respetado implica, también, estar atentos a lo que el bebé pide: marca la manera en que ayuda a su mamá para poder nacer. Si bien se trabaja para que se desarrolle de la manera más natural posible, en ocasiones, la indicación de una cesárea también es un nacimiento respetado, porque lo principal es que ese niño o niña nazcan sanos. Los bebés son los que van a marcar el pulso de cómo se desarrolla ese parto. A veces cuando no se da de manera natural es porque el bebé hace todo lo posible para no dañarse a sí mismo. Hay que conectarse con el hijo por nacer, él te dice cómo seguir. Gago, (2017).

Por ejemplo, el "plan de_parto" es un documento donde la mujer expresa sus preferencias, necesidades, deseos y expectativas sobre el proceso del parto y nacimiento. Esto incluye quiénes

\footnotetext{
403 Vol. 4, núm. 3, julio 2018, pp. 392-415 
deben estar presentes, dónde debe ocurrir, cómo le gustaría que sea el ambiente, qué posición(es) prefiere, qué métodos de alivio del dolor desea usar (o no), qué quisiera que se haga o deje de hacer a su recién nacido (corte del cordón umbilical tardío, baño), etc. Este documento se entrega antes del gran día en varias copias al médico, la matrona o partera, las personas que van a estar presentes y/o el personal de turno. Sería interesante explorar cómo esto influye en el parto y si se practica de forma masiva en los sectores público y privado. Johannsen, (2016).

El parto natural se puede hacer si la mujer lo requiere, es recomendable si ella lo quiere, lo que implica que no es un parto domiciliario, es un parto asistido en un centro de atención donde se deja la evolución espontánea, sin suero, ni anestesia, permitiendo su evolución natural. En cuanto a la posición de la madre, existe el parto vertical y el parto horizontal. Quienes han realizado partos verticales dicen que es más cómodo y más fácil, esto se puede lograr con mayor educación al respecto. La educación es una forma de fomentar el deseo de las madres y padres de tener un parto normal. Hott, (2016).

En términos generales, todas las definiciones se centran en un objetivo común: promover que el parto sea una experiencia positiva para la madre, independientemente del lugar en el que se desarrolle el nacimiento, aumentando el poder de decisión que la mujer tiene sobre este proceso, respetando sus valores, creencias y sentimientos, y reduciendo la excesiva medicalización y el número de intervenciones sistematizadas hoy en día desarrolladas, promoviendo la utilización de prácticas sanitarias individualizadas en función de las necesidades de cada mujer y basadas en la evidencia científica para garantizar el bienestar y la seguridad de la madre y el neonato, estableciéndose una conexión entre la matrona y la propia gestante. Biurrun, y Goberna, (2013), García, et al. (2010), Da Mata, Kakuda, (2014) y Goberna, et al. (2014).

\footnotetext{
404 Vol. 4, núm. 3, julio 2018, pp. 392-415 
El parto humanizado también contempla el uso de sillas obstétricas diseñadas de acuerdo a modelos existentes a lo largo de la historia. Ya en el Antiguo Egipto existían testimonios grabados de las mujeres arrodilladas sobre piedras o ladrillos sagrados, o bien acuclilladas, como antecedentes de las sillas paridoras que se usaron por centurias desde el mundo grecorromano. Una silla de baja altura permite a la madre estar en una posición casi acuclillada, favoreciéndole la apertura de sus piernas, con su pareja detrás con plena capacidad para sostenerla, con la posibilidad de tocarse y de mirarse. Y quienes los asisten, que compromete a todos los asistentes profesionales ubicados frente a frente, simétricamente, con las miradas cruzadas en un mismo plano, con las manos dispuestas al contacto directo y a la contención solicitada. Desde la visión de Burgo, el parto humanizado, sea o no con silla obstétrica, es un estímulo para un cambio ideológico profundo del dispositivo asistencial en los partos tradicionales. Burgo, (2004).

\section{Parto Intercultural Humanizado}

Para disminuir la mortalidad materna y neonatal, el MSP promueve políticas y acciones de salud para la atención del parto institucional. Esto incluye el desarrollo del Parto Tradicional Humanizado (PTH) en los servicios de salud del MSP: Se ha logrado generar un consenso entre los actores de salud - tanto la Dirección Provincial de Salud de la provincia de Morona Santiago, su Departamento de Salud Intercultural, como las organizaciones de las nacionalidades Shuar y Achuar, sobre el incremento de la cobertura de partos asistidos por personal calificado con respeto y adecuación de las formas culturales de atención. Esto dentro de un marco normativo de seguridad en la salud de las personas atendidas. UNICEF, (2009).

\footnotetext{
405 Vol. 4, núm. 3, julio 2018, pp. 392-415

Mariela G. Macías-Intriago; Javier I. Haro-Alvarado; Javier I. Haro-Alvarado; Gabriela L. GalarzaSoledispa; María del Carmen Quishpe-Molina; Brenda N. Triviño-Vera
} 
El parto tradicional humanizado se entiende como un desarrollo en la aplicación de los principios de la humanización del parto desde la interculturalidad, reconociendo las formas culturales de parto dentro de una institución pública de salud. UNICEF, (2009).

Para las nacionalidades Shuar las formas culturales más importantes de parto consisten tradicionalmente en lo siguiente:

- La posición vertical

- Asistencia del esposo

- Acompañamiento de otro familiar mujer

- Ambiente cálido

- Uso de hierbas (albahaca, piripri, hierba luisa) para regular las contracciones, para relajamiento

- Baño caliente y caldo de gallina criolla después del parto para relajamiento y fortalecimiento

- Entierro de la placenta

- El corte del cordón.

Roles tanto del esposo, esposa y la comunidad shuar en el PTH

El rol del esposo Shuar es muy importante en el parto domicilio en una comunidad amazónica. Tomando en cuenta que en muchos de los casos la única persona presente y en capacidad de ayudar a la mujer es el propio esposo, es el que debe aprender los cuidados prenatales, como asistir en el parto y los cuidados post natales. Los testimonios de los hombres Shuar indican 
alto conocimiento sobre el tema de parto y un compromiso fuerte para la seguridad de la mujer, feto y luego el recién nacido desde la cosmovisión Shuar. UNICEF, (2009).

Las madres de las mujeres embarazadas también juegan un rol importante. Deben estar pendientes para identificar cualquier riesgo de complicación. En la cultura Shuar no existen las parteras como un grupo identificable, sino que idealmente todos, hombres y mujeres, deberían saber cómo manejar el embarazo, parto y post parto según el rol que le corresponde. UNICEF, (2009).

Además de las tradiciones, se ha adoptado algunas prácticas del parto humanizado, como el uso de música y pelotas de relajación como opciones. El parto tradicional humanizado aumenta el control de la mujer sobre su cuerpo y respeta las formas individuales de cada mujer, sea mestiza o indígena. El principio del parto tradicional humanizado es un trato respetuoso que combina los principios del parto humanizado dentro de un contexto local de interculturalidad. UNICEF, (2009).

Beneficios de la "hora sagrada":

- Previene

la hipotermia

del

bebé.

Promueve la lactancia materna (tanto en partos naturales como cesáreas).

- Reduce el estrés del bebé y la ansiedad de la madre, mejorando el vínculo afectivo entre ambos.

- Contribuye a la estabilidad fisiológica del recién nacido.

- Está científicamente comprobado que el contacto precoz piel con piel estabiliza la respiración y la oxigenación del bebé, mantiene sus niveles de glucemia, estabiliza la presión arterial, disminuye el llanto e incrementa el estado de alerta tranquila. 
- Disminuye la presencia de síntomas depresivos post parto y fortalece la autoestima de la madre. OMS, (2018).

Los primeros sesenta a noventa minutos después del parto son trascendentes para la vida del bebé y su mamá. Todas las prácticas, procedimientos, intervenciones de rutina pueden postergarse hasta después de ese momento.

Si el parto no tuvo complicaciones, no es necesario bañar, medir, pesar, tomar la temperatura ni vacunar al recién nacido durante esos primeros minutos. Es importante también postergar el corte del cordón umbilical hasta que deje de latir, ya que el pasaje de flujo sanguíneo que se produce en ese momento es beneficioso para el sistema inmunológico del recién nacido. OMS, (2018).

Beneficios que trae el parto humanizado para la madre

El parto es la forma natural en que los bebés nacen y el cuerpo de las mujeres tiene mecanismos naturales para desencadenarlo y llevarlo a cabo. En el parto humanizado el personal de salud crea un entorno de respecto y tranquilidad, permite que la mujer se sienta empoderada como protagonista, e incentiva a la mujer a confiar en su cuerpo y su capacidad natural de dar a luz, evitar medicaciones contra el dolor y adoptar la posición en la cual ella se sienta más cómoda durante el trabajo de parto. Johannsen, (2016).

Sin embargo, en el modelo de atención que predomina hoy en día, la mujer llega a pasar varias horas acostada durante el trabajo de parto. En el momento del nacimiento, es llevada a una sala en donde debe adoptar una posición llamada "ginecológica", que a pesar de ser conveniente 
para los médicos, es incómoda para la mujer y puede aumentar el riesgo de contracturas musculares y desgarros de tejidos. Johannsen, (2016).

Uno de los médicos ecuatorianos que promueven proactivamente el parto humanizado es el Dr. Alex Escandón quien en 2016 recibió el premio en salud de la Organización Internacional para la Capacitación e Investigación Médica (IOCIM). En su opinión, desde el punto de vista médico y humano, la opción preferida antes de considerar intervenciones médicas debe darse las opciones lo más naturales posibles. Johannsen, (2016).

Durante una entrevista con el médico, el experto explicó "Si tienes una gripe o un dolor de garganta, ¿por qué hacerte una traqueotomía? La cirugía, la intervención y los medicamentos deben ser utilizados solo si los necesitas. Lo mismo aplica al parto, ¿por qué hacer una cesárea a una mujer que podría parir de manera natural?”. Johannsen, (2016).

Por otra parte, las cesáreas programadas que no resultan de una emergencia obstétrica durante el parto o una condición médica severa detectada previamente, en su gran mayoría son innecesarias porque carecen de una justificación médica estricta. Como toda cirugía, pueden tener complicaciones quirúrgicas (hemorragias) y posquirúrgicas (infecciones), además de prolongar el tiempo de estancia en el hospital, e incluso ocasionar problemas de cicatrización. Además de aumentar la probabilidad de cesáreas en los siguientes nacimientos. Johannsen, (2016).

A los beneficios físicos expuestos de un parto humanizado, se suma el bienestar emocional para la pareja cuando se crea un entorno de tranquilidad y respeto antes, durante y después del parto por parte de los profesionales y se permite la presencia y participación del padre (iQue emoción poder cortar el cordón umbilical de su bebé!, si desea hacerlo). Johannsen, (2016). 


\section{Beneficios que trae el parto humanizado para el bebé}

"La adopción de una posición vertical de la mujer durante el parto, favorece el flujo de oxígeno y nutrientes hacia la placenta, y por lo tanto, hacia el bebé, evitando el sufrimiento fetal. Otro ejemplo son las contracciones del trabajo de parto que, cuando el bebé está bien, ayudan a que se produzca la sustancia surfactante en los pulmones, la cual permite que el recién nacido tenga una respiración normal. Por eso, todas las cesáreas programadas sin razón médica aumentan el riesgo de problemas respiratorios del niño.” Johannsen, (2016).

La "hora sagrada". Se trata de un momento emotivo, sensible y único de encuentro, de reconocimiento, en el que el contacto precoz, piel con piel, tiene efectos positivos tanto en el corto como en el largo plazo. La madre experimenta una descarga de oxcitocina que favorece la instauración de la lactancia materna, ya que el bebé se encuentra especialmente receptivo, buscando una contención parecida a la que tenía dentro del útero. OMS, (2018).

.Además, también para el bebé hay beneficios emocionales difíciles de cuantificar: El contacto piel con piel inmediatamente después del parto entre el recién nacido y la madre, es importante porque representa el inicio del vínculo materno-infantil en la vida extrauterina. El vínculo materno-infantil tiene repercusiones en desarrollo psicológico, emocional y cognitivo del bebé, que son difíciles de medir y que a veces sólo se manifiestan en una etapa posterior del desarrollo. Además, este vínculo es un detonante importante de la lactancia materna. Johannsen, (2016).

En las palabras del experto: “Al nacer, en caso que el bebé salga de golpe y que se le separe de su madre, el niño ya perdió el primer contacto con la madre en los primeros momentos de vida en

\footnotetext{
\begin{tabular}{l|l} 
Vol. 4, núm. 3, julio 2018, pp. 392-415 \\
Mariela G. Macías-Intriago; Javie
\end{tabular}

Mariela G. Macías-Intriago; Javier I. Haro-Alvarado; Javier I. Haro-Alvarado; Gabriela L. GalarzaSoledispa; María del Carmen Quishpe-Molina; Brenda N. Triviño-Vera
} 
este medio ambiente tan diferente. El ser humano, como todos los mamíferos, apenas nace debe ser colocado junto a su madre. A diferencia de las consecuencias físicas, los riesgos emocionales de no hacerlo son difíciles de describir y cuantificar científicamente, aunque sabemos que el ser humano no es solo una suma de órganos, sino también una combinación de sentimientos y energía, con un montón de procesos que aún no entendemos al cien por ciento.” Johannsen, (2016).

El proceso del parto humanizado, brinda momentos emotivos para la madre como es el contacto piel a piel con su hijo. Tan pronto nace el bebé, el equipo médico lo adapta a su abdomen para que ella pueda sentirlo inmediatamente. También se presta asistencia a la madre para la lactancia de su bebé, lo cual estrecha el vínculo entre ambos y se favorece desde las primeras horas del nacimiento el traspaso de los nutrientes a su hijo para su crecimiento y desarrollo. Calderón, (2017).

Que cada vez más mujeres y parejas conozcan los beneficios físicos y emocionales de un parto humanizado, sería un primer paso importante para reducir la asimetría de información entre médicos y pacientes, reducir la tasa de cesáreas innecesarias y aumentar la demanda por partos naturales que sean respetuosos con las necesidades de la pareja y el bebé. Johannsen, (2016).

Una vez que la pareja tenga el conocimiento e interés, ¿qué debe hacer para tener un parto humanizado? Aquí una propuesta de cuatro pasos:

1. El más importante: Preguntar, preguntar y preguntar, sin miedo de hacer preguntas incomodas o insignificantes a médicos, establecimientos de salud y dentro de la red social personal. Conocer a detalle los servicios, opciones y el trato que ofrecen diferentes médicos o establecimientos es un paso clave para conocer la oferta local en la atención de partos.

\footnotetext{
411 Vol. 4, núm. 3, julio 2018, pp. 392-415

Mariela G. Macías-Intriago; Javier I. Haro-Alvarado; Javier I. Haro-Alvarado; Gabriela L. GalarzaSoledispa; María del Carmen Quishpe-Molina; Brenda N. Triviño-Vera
} 


\section{Tomar el tiempo necesario y actuar con anticipación para escoger el médico (o al} menos el establecimiento) con calma y cuidado, según las posibilidades respectivas en el sector privado o público aprovechando una de las consultas prenatales específicamente para planificar el parto con el médico, compartir un “plan de parto" o explicarle tus expectativas.

3. Conocer el lugar antes del parto, familiarizarse con la infraestructura y planificar un parto individualizado según sea posible (por ejemplo, llevar la música preferida, la pelota, el banquito o el aceite relajante, y lo más importante: una silla o colchón para la pareja, amiga y/o familiar de confianza que acompañe el parto).

\section{Optar por tener una doula, partera tradicional, amiga o pariente de confianza que} cumpla el rol de asistir a la mujer o pareja antes, durante y después del parto con asesoramiento no médico en los aspectos mentales, emocionales y logísticos de este gran evento pudiendo aliviar en gran medida el estrés, la ansiedad o inseguridad que exista.

Sea cual sea la decisión de cada embarazada o pareja, también depende de sus iniciativas proactivas el tener una experiencia satisfactoria en uno de los eventos más importantes de su vida, el parto. Por lo tanto, un parto humanizado no solo es nuestro derecho sino también nuestra responsabilidad. Johannsen, (2016).

\section{Conclusiones.}

Solo si la embarazada y su pareja están al tanto de sus opciones riesgos y beneficios ya sea para la madre como para el bebé tiene la importancia de lo que quieren y sobre todo, lo que no quieren solo así el parto puede ser humanizado, la mujer y la pareja conozcan los beneficios físicos y emocionales de el parto humanizado, sería un paso importante para dominar la desigualdad de

\footnotetext{
\begin{tabular}{l|l}
\hline 12 & Vol. 4, núm. 3, julio 2018, pp. 392-415 \\
Mariela G. Macías-Intriago; Javier I. Haro-Alvarado; Javier I. Haro-Alvarado; Gabriela L. Galarza-
\end{tabular} Soledispa; María del Carmen Quishpe-Molina; Brenda N. Triviño-Vera
} 
información entre médicos y pacientes, disminuir la tasa de cesáreas innecesarias y acrecentar la demanda por partos naturales que sean respetuosos con las necesidades de la pareja y el bebé. El parto humanizado es el parto natural o parto respetado donde se establece la comunicación afectuosa entre los padres y el equipo, donde se considera tanto los derechos de la madre como los del bebé a ser cuidado y asistido con todos los conocimientos que se poseen durante los años de experiencia, la asistencia del equipo en acompañar a la pareja que imagina el nacimiento de su bebé, respetando sus miedos y angustias haciendo todo lo mejor para el nacimiento del bebé. El parto es un instante transformador en la vida de una familia, y sobre todo en el de la mujer y el niño que está por nacer. Es un instante emotivo, sensible y único de encuentro, de reconocimiento, en el que el tierno contacto, piel con piel, tiene efectos tangibles tanto en el corto como en el largo plazo. La madre siente una descarga de oxcitocina que beneficia la instauración de la lactancia materna, ya que el bebé se encuentra especialmente receptivo, buscando una contención parecida a la que tenía dentro del útero.

\section{Bibliografía.}

Alonso, C. y Gerard, T., (2009), El parto humanizado como herramienta para la prevención de la mortalidad materna y la mejora de la salud materno - infantil. P. 95-100.

Alonso, C. y Gerard, T., (2009), El parto humanizado como herramienta para la prevención de la mortalidad materna y la mejora de la salud materno - infantil. P. 95-100.

Biurrun Garrido, A. y Goberna Tricas, J., (2013), La humanización del trabajo de parto: necesidad de definir el concepto, Revisión bibliográfica, Matronas Prof., 14(2):62-6.

Burgo, Carlos (2003), Litotomía en el parto: una práctica cuestionable. Clip-m. №1. Buenos Aires.

Burgo, Carlos, (2004), Parir con Pasión, Escenarios, escenas y protagonistas del parto y nacimiento, Longseller, Buenos Aires.

Calderón, Ivonne, (2017), Parto Humanizado: Más que un procedimiento médico, Maternidad y pediatría, Colsanitas.

\footnotetext{
413 Vol. 4, núm. 3, julio 2018, pp. 392-415

Mariela G. Macías-Intriago; Javier I. Haro-Alvarado; Javier I. Haro-Alvarado; Gabriela L. GalarzaSoledispa; María del Carmen Quishpe-Molina; Brenda N. Triviño-Vera
} 
Da Mata, J., Kakuda, A., (2014), Humanizing labor: experiences in the unified health system. Rev Min Enferm, 18(4):1011-5.

Fundación Juan Vives Suriá, (2012), El parto y nacimiento humanizado como derecho humano: un desafío para la transformación social, Defensoría del Pueblo, p.37.

García, E., Guillén, D., Acevedo, M., (2010), Humanismo durante la atención del trabajo de parto. Rev CONAMED, 15(3):152-4.

Gago, Nelly, (2017), La importancia del parto respetado, obstétrica de Halitus Instituto Médico.

Goberna, J., Palacio, A., Banús, M., Linares, S., Salas, D., (2008), Tecnología y humanización en la asistencia al nacimiento. La percepción de las mujeres. Matronas Prof., 9(1):5-10.

Hernández, A., Pascual, A., Bañó, A., Melero, M., Molina, M., (2014), Diferencias en el número de cesáreas en los partos que comienzan espontáneamente y en los inducidos. Rev. Esp. Salud Pública. 88(383-393).

Hott, (2016). La importancia del parto normal, ginecólogo del Hospital Base de Valdivia.

Johannsen, Julia, (2016), Popularizando nuevas tendencias o redescubriendo antiguas prácticas, especialista sénior en protección social en las oficinas del Banco Interamericano de Desarrollo en Ecuador.

Lange, Marie, (1998), Maternidad y Sexo, Paidós, Buenos Aires.

Maroto Navarro, G., García Calvente, M., Mateo Rodríguez, I., (2004), El reto de la maternidad en España, dificultades sociales y sanitarias, Gac Sanit., 18 Supl 2:13-23.

Prolhuna, Proyecto (2014), humanización de los cuidados en salud de las mujeres de la Maternidad Pública de Rocha, Manual de práctica clínica, Uruguay.

Martínez Galiano, J., (2011), Implantación del nuevo modelo de atención al parto. Actitud de los profesionales, Rev. Enferm. Ref. 3(5):65-71.

OMS, (2015), Declaración de la Organización Mundial de la Salud sobre tasas de cesárea, Ginebra: Organización Mundial de la Salud 2015, Available from.

http://www.who.int/reproductivehealth/publications/maternal_perinatal_health/cs-statement/es/

OMS, (1985), Declaración de Fortaleza. Recomendaciones de la OMS sobre el nacimiento, Organización Mundial de la Salud, Lancet. 1985, 2:436-7.

OMS, (1996), Organización Mundial de la Salud. Cuidados en el parto normal: Una guía práctica, Ginebra.

OMS, (2018). Hablemos de parto humanizado y la "hora sagrada", Organización Mundial de la Salud. 
Silva, R., Soares, M., Muniz, R., Andrade, F., Torres, A., Gomes, V., (2011), La concepción de los profesionales de salud sobre el parto humanizado en la adolescencia Enfermería Global, Vol. $10, \mathrm{~N}^{\circ} 4$, pp. 331-340.

UNICEF, (2009), Salud y Nutrición - Parto Intercultural Humanizado, Ecuador.

https://www.unicef.org/ecuador/health_nutrition_16853.htm

Vela, G., (2015), Percepción de la paciente sobre la atención del parto humanizado en el Servicio de Centro Obstétrico del Instituto Nacional Materno Perinatal, durante el periodo marzo-abril de 2015, Tesis de Licenciatura Universidad Nacional Mayor de San Marcos. Facultad de Medicina de San Fernando, Lima-Perú.

Whitford, H. y Hillan, E., (1998), Women's perceptions of birth plans, Midwifery, 14(4):248-53. 